\title{
BUILDUP OF MAGNETIC SHEAR AND FREE ENERGY DURING FLUX EMERGENCE AND CANCELLATION
}

\author{
FAng FAnG $^{1}$, Ward Manchester IV ${ }^{1}$, William P. AbBetT ${ }^{2}$, And Bart VAn Der Holst $^{1}$ \\ ${ }^{1}$ Department of Atmospheric, Oceanic and Space Sciences, University of Michigan, Ann Arbor, MI 48109, USA \\ ${ }^{2}$ Space Sciences Laboratory, University of California, Berkeley, CA 94720, USA \\ Received 2012 March 23; accepted 2012 May 14; published 2012 June 29
}

\begin{abstract}
We examine a simulation of flux emergence and cancellation, which shows a complex sequence of processes that accumulate free magnetic energy in the solar corona essential for the eruptive events such as coronal mass ejections, filament eruptions, and flares. The flow velocity at the surface and in the corona shows a consistent shearing pattern along the polarity inversion line (PIL), which together with the rotation of the magnetic polarities, builds up the magnetic shear. Tether-cutting reconnection above the PIL then produces longer sheared magnetic field lines that extend higher into the corona, where a sigmoidal structure forms. Most significantly, reconnection and upward-energy-flux transfer are found to occur even as magnetic flux is submerging and appears to cancel at the photosphere. A comparison of the simulated coronal field with the corresponding coronal potential field graphically shows the development of non-potential fields during the emergence of the magnetic flux and formation of sunspots.
\end{abstract}

Key words: magnetohydrodynamics (MHD) - Sun: atmosphere - Sun: photosphere

Online-only material: color figures

\section{INTRODUCTION}

Regions of intense solar magnetic fields such as sunspots and active regions are known to exhibit energetic outbursts that are manifest in many forms, such as coronal mass ejections (CMEs), filament eruptions, and flares (e.g., Forbes 2000; Low 2001; Forbes et al. 2006; Gopalswamy 2006; Schrijver 2009). In the most dramatic form, magnetic fields release energy up to $10^{32} \mathrm{erg}$, and in the process expel $10^{15} \mathrm{~g}$ of plasma and $10^{20} \mathrm{Mx}$ of magnetic flux into interplanetary space (Bieber \& Rust 1995) at speeds that can reach $3000 \mathrm{~km} \mathrm{~s}^{-1}$ (e.g., Gosling et al. 1990; St. Cyr et al. 2000; Zhang et al. 2004). Although less energetic, large-scale regions of relatively weak magnetic fields can also erupt giving rise to streamer blowouts and the expulsion of quiescent filaments into the solar wind (e.g., Hundhausen 1993; Lynch et al. 2010).

In order to produce such eruptions, the magnetic field must possess free magnetic energy, which requires that the field is in a non-potential configuration with electric currents passing through the photosphere and into the corona. In general, the field lines of the non-potential force-free fields are stretched, expanded, and intertwined compared to the equivalent potential field mapping to the photosphere. Such force-free fields in the solar corona have historically been taken to be of two forms: arcades, in which the footpoints have been sheared to produce a strong field component parallel to the polarity inversion line (PIL), or flux ropes, in which the magnetic field is twisted about a central axis. This dichotomy of field configurations naturally leads to two broad groups of theoretical and numerical models of CMEs.

In the case of magnetic arcades, initial configurations are typically taken to be line-tied to the lower boundary and subsequently energized with prescribed shear flows, which cause the arcade to expand and then erupt (e.g., Steinolfson 1991; Mikic \& Linker 1994; Amari et al. 1996; Guo \& Wu 1998). Another variant, the so-called breakout model, involves a system of quadrupolar fields with a system of three arcades. The field reverses direction over the central arcade allowing magnetic reconnection to release the central arcade (e.g., Antiochos et al.
1999; Lynch et al. 2004, 2008; van der Holst et al. 2009). In all of these cases, magnetic flux ropes form during the eruption process and are expelled from the corona. For those models which assume that flux ropes exist prior to the eruption, the flux ropes are assumed to reside within an arcade where the outward-directed Lorentz force of the rope (hoop force) is balanced by magnetic tension of the surrounding arcade (e.g., Forbes \& Isenberg 1991; Low 1994; Titov \& Démoulin 1999). The central question with flux rope models is how the force imbalance occurs and results in eruption. Flux ropes have been energized by the application of rotational motions at the rope footpoints (e.g., Tokman \& Bellan 2002; Rachmeler et al. 2009) that twist up the field. Flux ropes have also been forced to emerge into the corona by bodily advecting them through the lower boundary of the computational domain (e.g., Török \& Kliem 2005; Fan 2005). In the case of Amari et al. (2003), flux cancellation at the PIL forms a flux rope within a sheared magnetic arcade. Following these formation processes, the flux rope may lose equilibrium and erupt from several instabilities including imbalance of magnetic forces, which is followed by magnetic reconnection (Forbes \& Isenberg 1991; Amari et al. 2003; Roussev et al. 2004), torus instability (Török \& Kliem 2005), or kink instability (Sturrock et al. 2001; Fan 2005).

Plentiful observations provide evidence for both arcades and flux ropes as the coronal field structures for CMEs. Sheared magnetic fields are measured directly at the photosphere with vector magnetographs (e.g., Hagyard et al. 1984; Zirin \& Wang 1993; Falconer 2001; Yang et al. 2004; Liu et al. 2005), where the field is found to run nearly parallel to the PIL. More recent analysis by Schrijver et al. (2005) found that shear flows associated with flux emergence drove enhanced flaring. Similarly, active region $\mathrm{CME}$ productivity is also strongly correlated with magnetic shear, as shown by Falconer et al. (2002, 2006). There is also evidence suggesting that flux ropes may bodily emerge through the photosphere to reside in the corona. The twist of the emerging flux tube is characterized by the presence of elongated magnetic polarities, i.e., magnetic tongues in longitudinal magnetograms (Luoni et al. 2011). Leka \& Steiner (2001) examined a time series of vector magnetograms of emerging magnetic flux 
and found that magnetic fields passing through the photosphere are already twisted. Similarly, Lites et al. (1995) examined a time series of photospheric vector magnetograms, which they found could be fitted by the emergence of a spherically shaped magnetic flux rope. Lites (2005) shows an example of the photospheric vector magnetic field and chromospheric structure seen in the $\mathrm{H} \alpha$ line, which suggest the presence of a coronal flux rope associated with a prominence.

Magnetohydrodynamic (MHD) numerical simulations may provide new insights into our understanding of the physical processes that build up the free magnetic energy necessary for solar eruptions. Global scale simulations address how magnetic fields generated at the tachocline pass through the convection zone and have explained and reproduced many large-scale aspects of sunspots (e.g., Spruit \& van Ballegooijen 1982; Abbett et al. 2001; Fan 2008). However, because of the anelastic approximation, these models can only extend up to a height of $20 \mathrm{Mm}$ below the photosphere. Above this depth, fully compressible MHD models have been employed to simulate the emergence of magnetic fields from the near-surface convection zone into the lower corona. Early work demonstrated the basic processes by which fields emerge to form bipolar active regions (e.g., Shibata et al. 1989; Matsumoto et al. 1993). These simulations were followed by cases (e.g., Manchester 2001; Fan 2001; Magara \& Longcope 2003) which showed that the Lorentz force, arising when fields expand into the highly stratified solar atmosphere, drives shear flows along the PIL. The shear flows then transport magnetic energy into the coronal portion of the flux rope which then expands and drive its eruption (Manchester et al. 2004). This shearing process is suggested as the energy source for CMEs and flares (Manchester 2007, 2008). Galsgaard et al. (2007), Archontis \& Török (2008), and MacTaggart \& Hood (2009) produce fast CME-like eruptions by also invoking magnetic reconnection with emerging flux ropes. Fan (2008) simulated flux rope emergence that exhibited sunspot rotation driven by a torsional form of the Lorentz force, which twisted up the coronal portion of the field as predicted by Parker (1977). However, in this case, no eruptive behavior was found.

Recently, computing power has reached a level that has allowed the development of realistic solar models, including radiative and thermodynamic processes necessary to simulate convection in conjunction with the upper atmosphere. Stein \& Nordlund (2006), Abbett (2007), Rempel et al. (2009), Cheung et al. (2010), Kitiashvili et al. (2010), and Rempel (2011) emphasize the importance of the interaction between magnetic fields and convective motions. Stein \& Nordlund (2006) and Abbett (2007) have each addressed quiet-Sun magnetoconvection, while Kitiashvili et al. (2010) report the critical role of strong vortical downdrafts around small magnetic structures in the formation of large-scale structures. The work of Rempel et al. (2009) and Cheung et al. (2010) treats the formation and evolution of sunspots, including the Evershed flow. Fang et al. (2010, 2012) address the emergence of magnetic flux ropes from a turbulent convection zone into the corona and found both shearing and rotational flows driven by the Lorentz force. These horizontal flows were found to dominate the energy transport from the convection zone into the corona. Of particular significance, the simulation of Fang et al. (2012) exhibited a case of large-scale flux cancellation, a phenomenon strongly associated with CME initiation (Subramanian \& Dere 2001). Here, we examine the flux cancellation in conjunction with energy transport from the convection zone to the corona.
The paper is organized in the following way: Section 2 describes the numerical simulation; Section 3 studies the buildup of magnetic shear in a flux cancellation event, followed by analysis on the free energy in the corona in Section 4. The energy transfer at the photosphere is discussed in Section 5. Section 6 summarizes our conclusions and discussion.

\section{NUMERICAL SIMULATION}

To simulate the flux emergence in the convection zone, we first generate a relaxed solar atmosphere of dimensions $30 \times 30 \times 42 \mathrm{Mm}^{3}$, with the photosphere located at $Z=0 \mathrm{Mm}$. Our model solves the MHD equations in Block-Adaptive Tree Solar-wind Roe Upwind Scheme (BAT-S-RUS) with additional energy source terms to describe thermodynamic processes in the solar atmosphere, i.e., radiative cooling at the photosphere and in the corona and coronal heating (Abbett 2007; Fang et al. 2010). Meanwhile, implementation of the non-ideal tabular equationof-state (Rogers 2000) provides a more accurate description of the partially ionized plasma in the convection zone. We use periodic horizontal, closed upper boundary conditions, and fix the density and temperature values at the lower boundary while setting the vertical momentum to be reflective.

Taking advantage of the adaptive grid in BAT-S-RUS, our model produces a relaxed convection zone with a depth of $20 \mathrm{Mm}$, with an overlying photosphere of $0.45 \mathrm{Mm}$ thickness and a corona of $20 \mathrm{Mm}$, in a Cartesian domain. The vertical stratification of the atmosphere in our simulation domain is shown in Figure 1 in Fang et al. (2012). We then carry out a simulation of the emergence of a buoyant, initially stationary, horizontal flux rope inserted at $Z=-10 \mathrm{Mm}$, shown in Figure 2 in Fang et al. (2012). Interaction of the rising flux rope with large-scale convective motion produces the bipolar structure of the flux rope, with the convective downflows fixing the two ends of the emerged section of the flux rope in the convection zone, illustrated in Figure 5 in Fang et al. (2012). Near-surface small-scale convection produces the magnetic polarities by coalescence and intensifies the strength of the magnetic flux. A small active region forms on the photosphere, seen in Figure 6 of Fang et al. (2012), exhibiting strong interaction of the magnetic flux and the surface flows, i.e., the shearing and converging motions, separation and rotation of the magnetic polarities. In the presence of these flows, a flux cancellation event takes place at time $t=05: 00: 00$, shown by Figure 6 of Fang et al. (2012), during which $10 \%$ of the total photospheric unsigned flux is canceled. During the flux emergence, horizontal motions dominate the energy transfer to the corona, while vertical flows transport energy back into the convection zone. Details on the dynamics of the emerging flux rope are discussed in Fang et al. (2012).

\section{A CASE OF FLUX CANCELLATION}

Here, we examine a region of magnetic flux cancellation at the photosphere in our simulation, which is also associated with high magnetic shear. Flux cancellation of this form is particularly significant as it has been observed to be associated with flares (Martin et al. 1985) and CMEs (Subramanian \& Dere 2001) in active regions. A clear example is observed in AR 10977, where reconnection at the PIL during flux cancellation forms a highly sheared arcade and sigmoidal structure that subsequently reforms after eruption (Green et al. 2011). Li et al. (2004) found converging flows consistent with flux cancellation in decaying and young active regions producing CMEs. Inspired by similar observations, converging motion and 


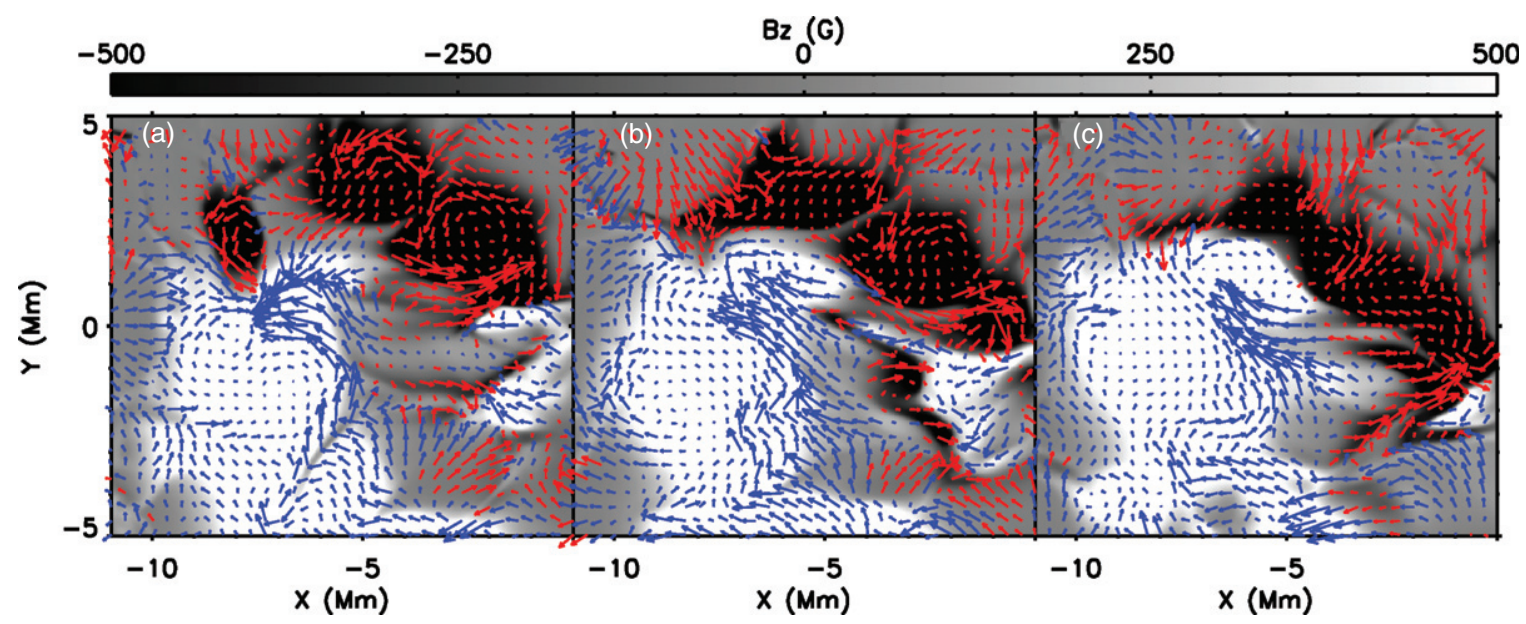

Figure 1. Photospheric structure of $B_{z}$ in the area of flux cancellation at time $t=5: 00: 00$ (a), 5:10:00 (b), and 5:22:00 (c). Blue and red arrows show the horizontal velocity in positive and negative magnetic polarities, respectively. Shear flows are most apparent at time $t=5: 10: 00$.

(A color version of this figure is available in the online journal.)
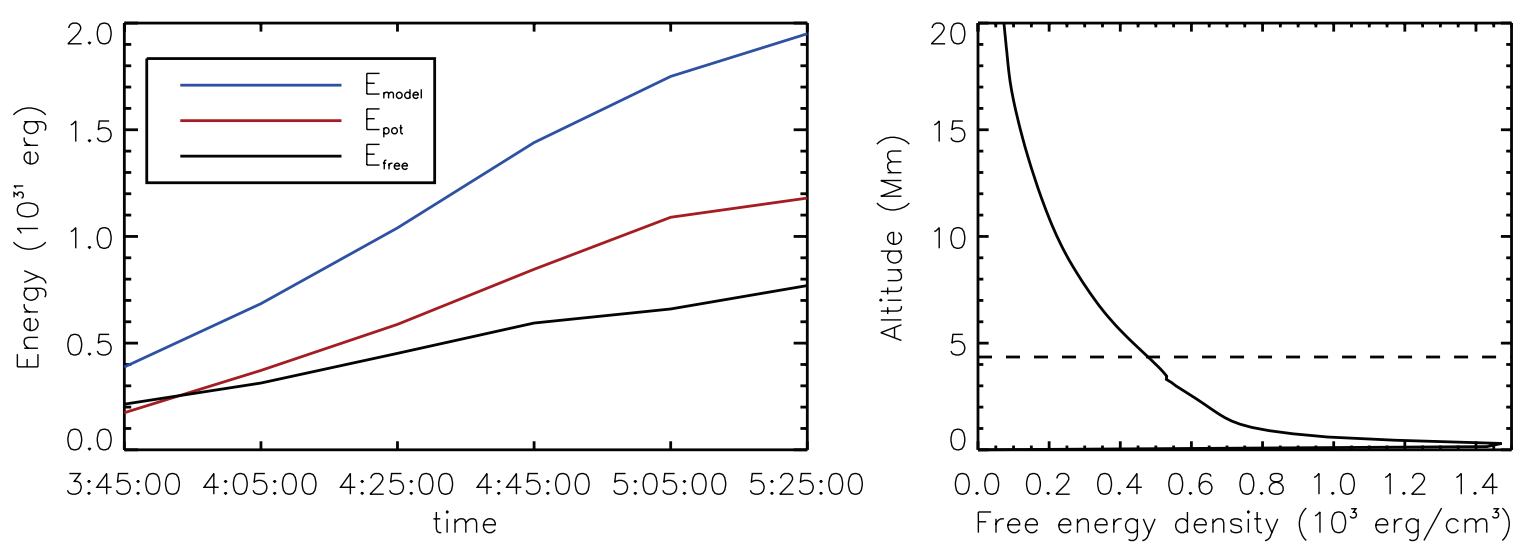

Figure 2. Left: temporal evolution of the total (blue), potential (red), and free (black) magnetic energy. Right: vertical distribution of free energy density at time $t=$ 05:05:00. The dashed line indicates the altitude up to where 50\% of the coronal free energy is stored.

(A color version of this figure is available in the online journal.)

flux cancellation have been imposed as boundary conditions in MHD simulations to produce eruptions (Linker et al. 2003; Amari et al. 2003).

As it is observed on the Sun, flux cancellation is very ambiguous as it may be caused by submergence of $\Omega$-loops, emergence of U-loops, or magnetic cancellation by some dissipative process. Our simulation provides a unique opportunity to fully understand an example of flux cancellation in a way that is not possible through observations alone. We find flux cancellation occurs spontaneously between opposite polarities that are driven together by the convective flows near the photosphere. The temporal evolution of cancellation is shown in Figures 1(a)-(c), which show a zoom-in view of the relevant area, with background color showing the photospheric $B_{z}$ field. The horizontal motions in positive and negative polarities are shown by blue and red arrows, respectively. The flux cancellation event starts at time $t=05: 00: 00$, lasts for $0.5 \mathrm{hr}$, and the total amount of canceled flux approaches $1 \times 10^{20} \mathrm{Mx}$, which is $10 \%$ of the total unsigned flux on the photosphere. It is remarkable that during the process of flux cancellation, the coronal free energy (shown in the left panel of Figure 2) is still increasing even though the photospheric magnetic flux is decreasing.

To understand this process, we take a close examination of the velocity fields in the area of flux cancellation prior to, during, and in the later phase of the event, as shown by Figures 1(a)-(c), respectively. In Figure 1(a), the two polarities are pushed together by the horizontal converging motion at the two sides of the PIL. The flow pattern at the PIL is mostly converging and forms a very narrow PIL at a later time illustrated in Figure 1(b). Figure 1(b) shows a clear shearing motion at the PIL, with two polarities running in opposite directions. The strong shear flow at the PIL is driven by the Lorentz force, and takes place during the flux cancellation as the magnetic field expands in the corona as shown by the blue colored field lines in Figures 3(a) and (b). Figure 4(a) shows the horizontal Lorentz force at time $t=$ 05:01:00 with arrows, from which we find a clear pattern of the force running in opposite directions across the PIL in the area of flux cancellation. At time $t=5: 22: 00$, toward the end of the flux cancellation, the shearing motion is weakened and the flow pattern becomes dominated by the converging motion again, as shown in Figure 1(c). The weak-strong-weak shearing motion in the flux cancellation event is also shown by observations $\mathrm{Su}$ et al. 2007) during flares where footprints of the magnetic fields present the same pattern of motion.

Figures 3(a)-(c) show the configuration of the magnetic fields in the area of flux cancellation at time $t=05: 05: 00$. Figure 3(a) provides the top view of the fields, and we find that instead of short loops perpendicular to the PIL, as is the case for potential fields, the field lines rather are elongated parallel to the PIL. Furthermore, the fields are more parallel to the PIL as they 

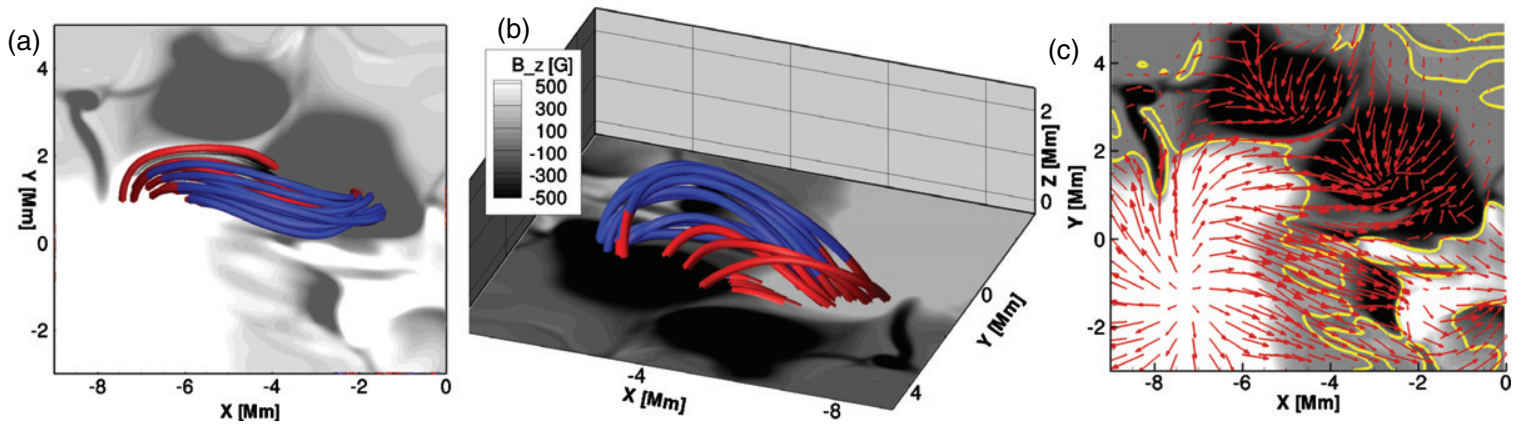

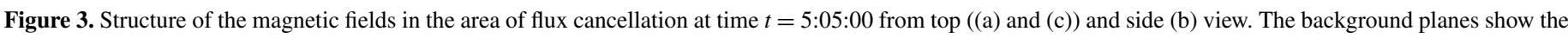

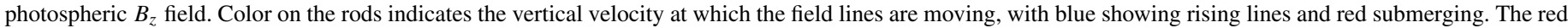
arrows in panel (c) represent the horizontal magnetic field, with yellow line showing PIL.

(A color version of this figure is available in the online journal.)
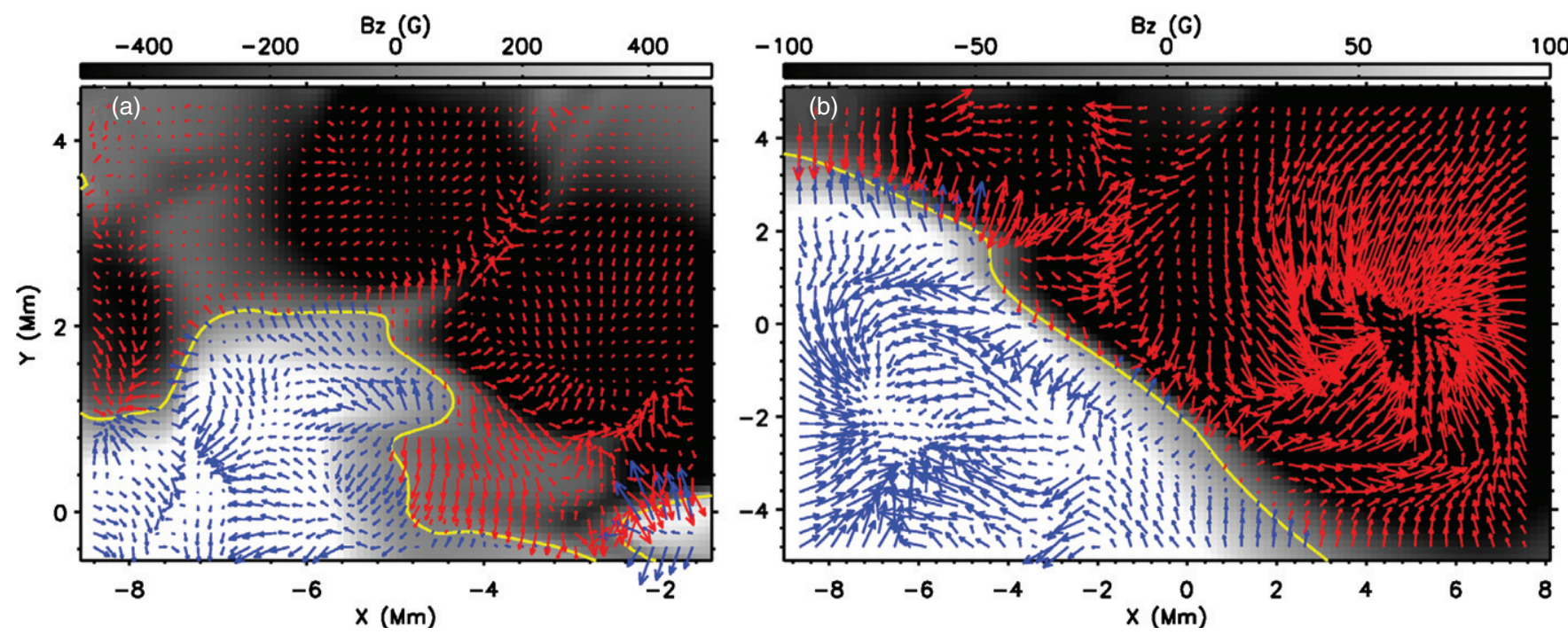

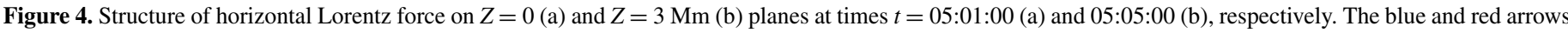

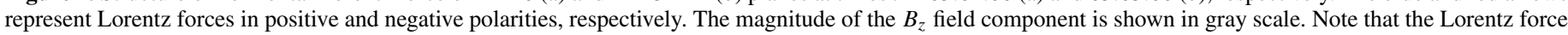
reverses direction across the PIL driving the shear flow and forms a spiral pattern in the negative polarity driving a rotating flow.

(A color version of this figure is available in the online journal.)

become closer to it, as shown by the red arrows in Figure 3(c). At the photosphere, the magnetic fields are submerging to the convection zone at the PIL, as shown by the red rods in Figure 3(b). The concentration of both the magnetic and velocity shear along the PIL demonstrates that the elongation of the field lines is the result of shearing motion, which is further enhanced by the converging motion at the PIL (Martens \& Zwaan 2001). These converging motions also give rise to occurrence of tether-cutting reconnection close to the photosphere in the highly sheared fields as proposed by Moore et al. (2001) as an explanation for solar eruptive events. Figure 1 of Moore et al. (2001) envisioned reconnection inside a highly sheared core field, which would produce longer, nearly horizontal field lines higher in the corona, while a system of short unsheared loops would form at the base of the arcade. This flux transfer process accumulates the free energy of the sheared field higher in the corona while at the same time reducing the magnetic tension by detaching the system from the photosphere. The rise of the arcade naturally leads to necking off of the fields, which in turn promotes more reconnection. Unabated, the runaway reconnection provides a mechanism for the initialization and growth of explosive events. With our simulation, we can fully examine the way in which submergence and reconnection alter the field line geometry in the tether-cutting process. A three- dimensional (3D) view of the magnetic fields combined with the flow pattern provides a complete picture of the effects of the internal reconnection. Figure 3(b) illustrates the structure of the magnetic field lines colored by the vertical component of the velocity orthogonal to the field lines. Blue indicates upflows and red downflows. We find two groups of magnetic field lines formed during tether-cutting reconnection, with one rising up into the corona and the other, shorter loops submerging into the convection zone. At the photosphere, the submergence of the shorter loops, as shown by the red rods right above the PIL, results in the decreased unsigned flux observed at the surface. Magnetic shear accumulates along the long, rising loops, forming a highly sheared arcade.

To study the energy transport into the corona during flux cancellation, we examine the Poynting flux at $Z=3 \mathrm{Mm}$ associated with the vertical motions. At the surface, the vertical motion transports the energy back into the convection zone due to the concentration of the magnetic flux in downflows. In the corona, the acceleration of magnetic fields after reconnection plays a very important role in the energy flux, shown by Figure 5(e). At the PIL in the corona, we observe a strong energy input associated with the rising motion of the fields. The rising motion here in the corona, however, is different from the emerging motion at the photosphere. At the photosphere, 

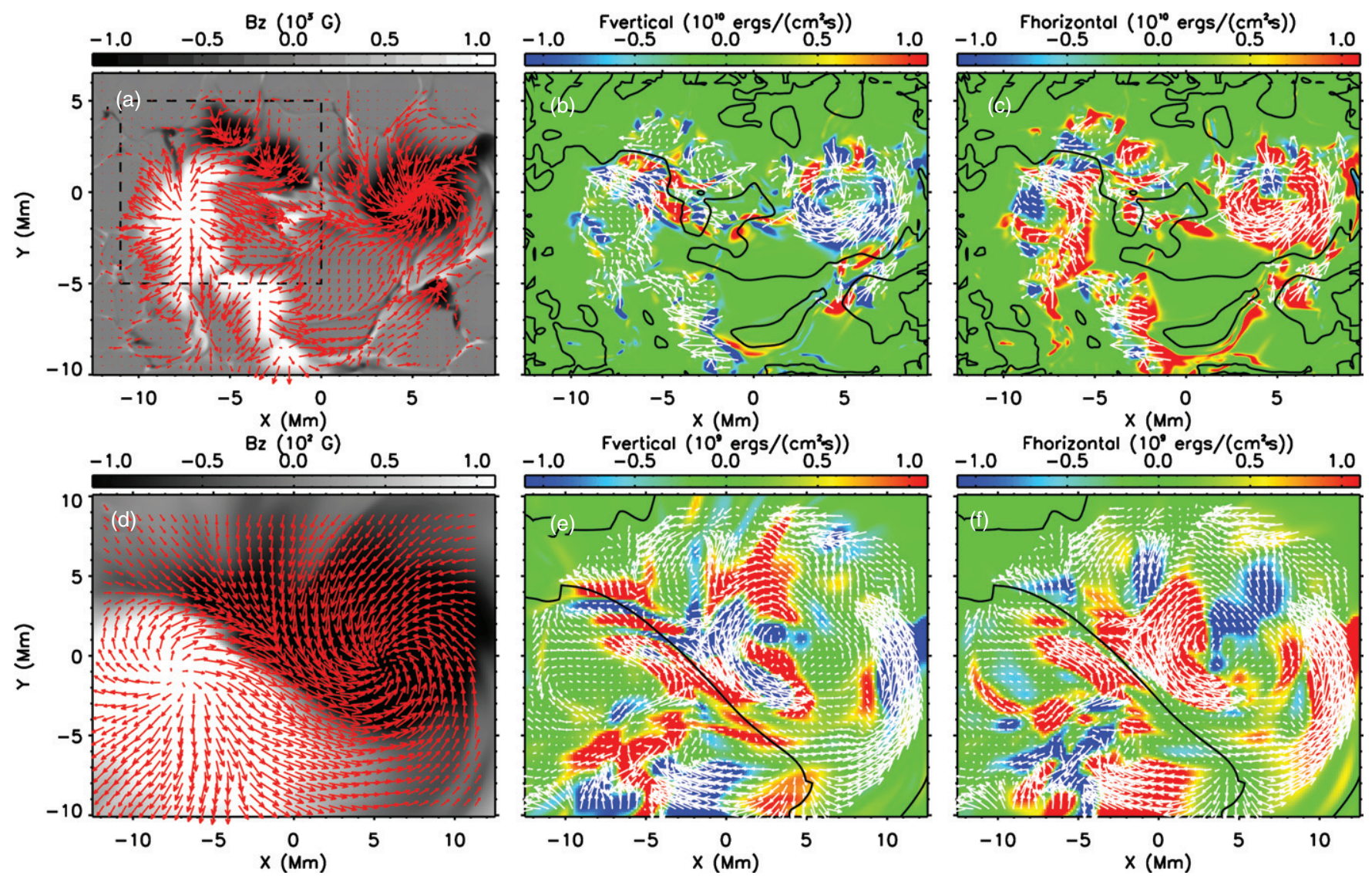

Figure 5. Structure of $B_{z}\left((\mathrm{a})\right.$ and (d)), $F_{\text {vertical }}\left((\mathrm{b})\right.$ and (e)), and $F_{\text {emerge }}((\mathrm{c})$ and (f)) fields at the photosphere ((a), (b), and (c)) and at $Z=3 \mathrm{Mm}$ in the corona ((d), (e), and (f)) at time $t=05: 05: 00$. PIL is shown by the black line. Red arrows in panels (a) and (d) represent the horizontal magnetic fields, and white arrows in (b), (c), (e), and (f) show the horizontal velocity fields.

(A color version of this figure is available in the online journal.)

the emergence is caused by the expansion and buoyancy of the flux rope as well as convective upflows. However, in the corona, the tether-cutting reconnection forms longer loops possessing less magnetic tension, resulting in a magnetic pressure gradient force accelerating the magnetic fields upward.

Besides reconnection and shear flows, rotation of the magnetic polarities also contributes to the accumulation of magnetic shear by moving the footpoints in opposite directions at the PIL. Figure 6 shows the geometry of two groups of coronal magnetic field lines: purple overlying lines with apex higher than $5 \mathrm{Mm}$ and green lines lower than $5 \mathrm{Mm}$. Horizontal velocity fields at $Z=3 \mathrm{Mm}$, represented by the arrows, show a rotation pattern on the negative polarity, which is also seen in Figure 5(e). The rotation complements the shearing motion at the PIL, in the sense that both shear and rotation contribute to plasma motions parallel to the PIL. However the flow patterns are also quite distinct, occurring at different scales and at different times. The shear flows are localized to the PIL and persist even when there is not a coherent rotation pattern in the magnetic polarities. When rotation is present, the shear flow tends to be greater in magnitude and will be shown graphically to dominate the energy transport into the corona.

The combination of the two motions creates a highly sheared arcade at the PIL, shown by the green rods in Figure 6, and rotation produces the twisted field structure at the far ends of the emerged fields, shown by purple rods. Both motions are driven by the Lorentz force, as shown by the arrows in Figure 4(b). At the PIL, the horizontal Lorentz force runs in the opposite direction, consistent with the directions of the shearing motion. In the negative polarity, the Lorentz force rotates in the same way as the rotation of the polarity. The sigmoidal structure at the PIL is maintained by the consistent shearing and rotating motions in our simulation. The relative contribution of shear flows compared to rotation can be seen in the geometry of the coronal fields at the PIL. Here, the field is dominated by an arcade structure instead of a twisted flux rope. Most of the twist is located at the outer periphery of the two polarities. Even in the most twisted field lines, there is less than a full turn of the field around the axis of either polarity. The absence of the twisted flux rope may be explained by two facts: the axis of the initial magnetic flux rope remains in the convection zone, and there are not enough twisting motions on the surface to reproduce such a twisted structure after emergence. The emerged fields are sheared and elongated, forming the arcade structure over the PIL.

\section{NON-POTENTIALITY OF THE MAGNETIC FIELD}

During our simulation, a significant amount of energy, up to $2 \times 10^{31} \mathrm{erg}$, is transferred into the corona over $5 \mathrm{hr}$ of evolution. The question is then: how much of the magnetic energy is in a free form, which is necessary for solar eruptive events? To investigate the evolution of magnetic free energy, we compare the coronal magnetic fields in our simulation with potential fields extrapolated from the photospheric boundary conditions. Figure 7 shows the temporal evolution of the model fields 


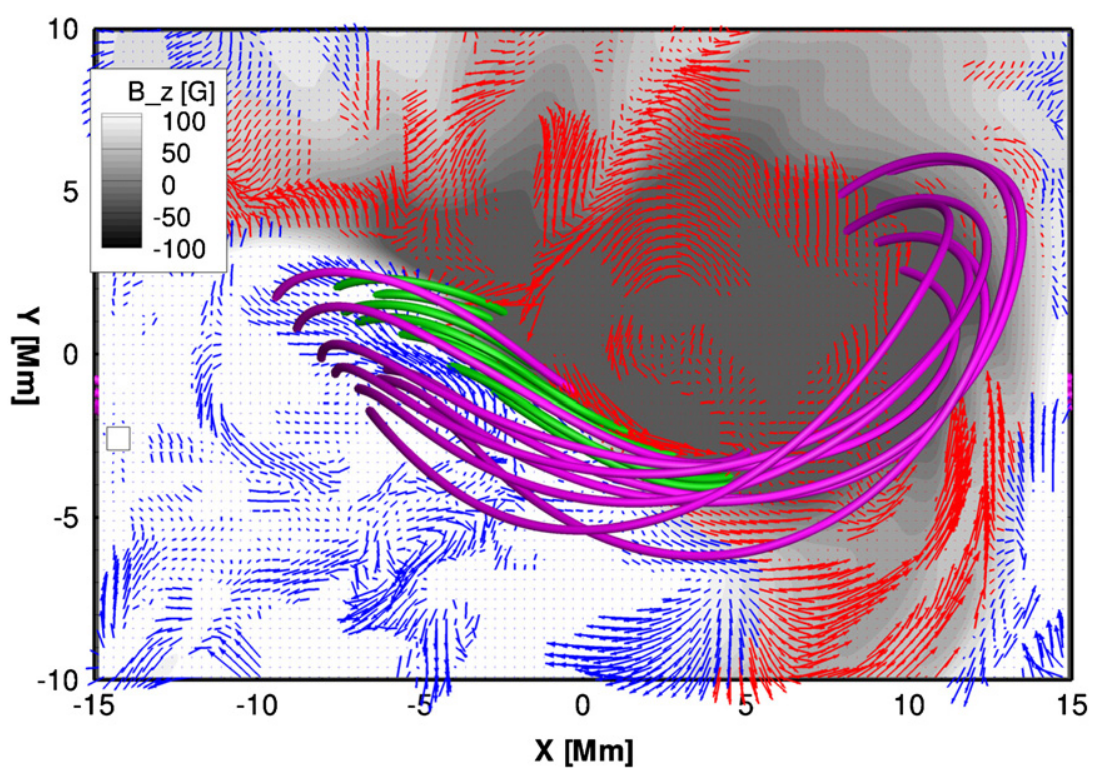

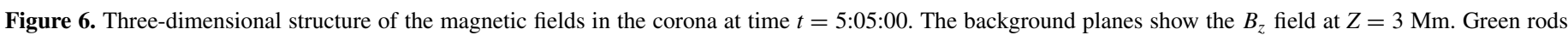

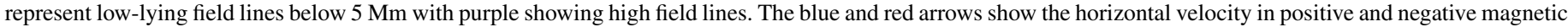

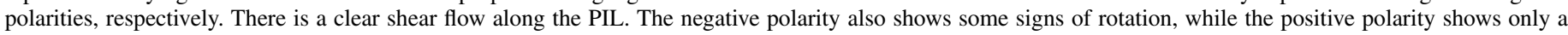
coherent shear flow. The magnetic field lines clearly reflect the flow pattern.

(A color version of this figure is available in the online journal.)

(blue) and the extrapolated potential fields (red). Comparison between the two fields clearly shows the buildup of magnetic shear along the field lines during the process of flux emergence. At time $t=04: 05: 00$, shown by the upper-left panel in Figure 7, the magnetic flux concentrates in two polarities, with most of the field lines running perpendicular to the PIL. The energy transfer into the corona occurs as the magnetic field lines become elongated and sheared along the PIL. The lower-right panel in Figure 7 shows the field structure at time $t=05: 05: 00$, in which the field lines are sheared along the PIL and compressed into the lower atmosphere while the overlying fields remain almost potential. The simulated configuration of the magnetic fields in our domain is consistent with observations and simulations of the magnetic fields before solar eruptions (Schrijver et al. 2008; Leake et al. 2010). The overlying fields confine and compress the sheared core in the lower atmosphere, which may be destabilized and give rise to sudden release of magnetic free energy.

Here, we calculate the free energy for the simulation by integrating the magnetic energy for the model and subtracting the energy of the potential field. The results are shown in the left panel of Figure 2, where the temporal evolution of model, potential, and free energy is plotted with blue, red, and black lines, respectively. Beginning at time $t=04: 05: 00$, the amount of free energy doubles within $1 \mathrm{hr}$ (the period shown by Figure 7), such that the coronal free energy approaches $7 \times 10^{30} \mathrm{erg}$ while the total energy is $1.7 \times 10^{31} \mathrm{erg}$. At this time, the free energy contributes to $40 \%$ of the total energy. Meanwhile, the vertical stratification of the free magnetic energy is shown in the right panel of Figure 2. The line plot shows a strong tendency of concentrating free energy in the lower atmosphere, with $50 \%$ located below $Z=4 \mathrm{Mm}$, shown by the dashed line. Figures 8(a) and (b) show the angle between the current and the magnetic field and the structure of $\alpha$ (the ratio of $|4 \pi \mathbf{j}|$ and $|\mathbf{B}|$ when the angle between them is less than $10^{\circ}$ ) in the $Y=0$ plane, respectively. The non-force-free magnetic fields, as shown in Figure 8(a), mainly reside in the convection zone, the core sheared field close to the surface and the negative polarity where the rotation forms a twisted magnetic structure (see Figure 7). Note that $\alpha$ tends to be maximized low down the center of the arcade structure and then falls off with distance from the PIL.

\section{POYNTING FLUXES}

To separate the energy transfer associated with vertical and horizontal flows, we decompose the Poynting flux into two components:

$$
\begin{gathered}
F_{\text {horizontal }}=-\frac{1}{4 \pi}\left(B_{x} u_{x}+B_{y} u_{y}\right) B_{z}, \\
F_{\text {vertical }}=\frac{1}{4 \pi}\left(B_{x}^{2}+B_{y}^{2}\right) u_{z} .
\end{gathered}
$$

$F_{\text {vertical }}$ indicates the energy transported by the vertical motion, while $F_{\text {horizontal }}$ represents the energy flux due to the horizontal motions, which include, at the photosphere, the separation of small dipoles, rotation, and the shearing flow along the PIL. Figures 5(b) and (c) show the structures of $F_{\text {vertical }}$ and $F_{\text {horizontal }}$, respectively, with the arrows representing the horizontal velocity fields. It is clear that in regions with rotation or shearing there is a strong energy input into the corona. Comparison of the structures of $F_{\text {vertical }}$ and $F_{\text {horizontal }}$ suggests that horizontal motion dominates in the energy transfer during the current phase of emergence, while vertical motion transfers energy back into the convection zone, due to the concentration of magnetic flux in downflow regions (Fang et al. 2010, 2012). Vertical emerging motion dominates at the very beginning of the flux emergence, and later gives way to horizontal motions, a pattern found earlier by Magara \& Longcope (2003) and Manchester et al. (2004).

At the photosphere, the magnetic field is very highly structured by convection. In particular, the flow patterns and Poynting fluxes can be difficult to interpret, with a large component of 


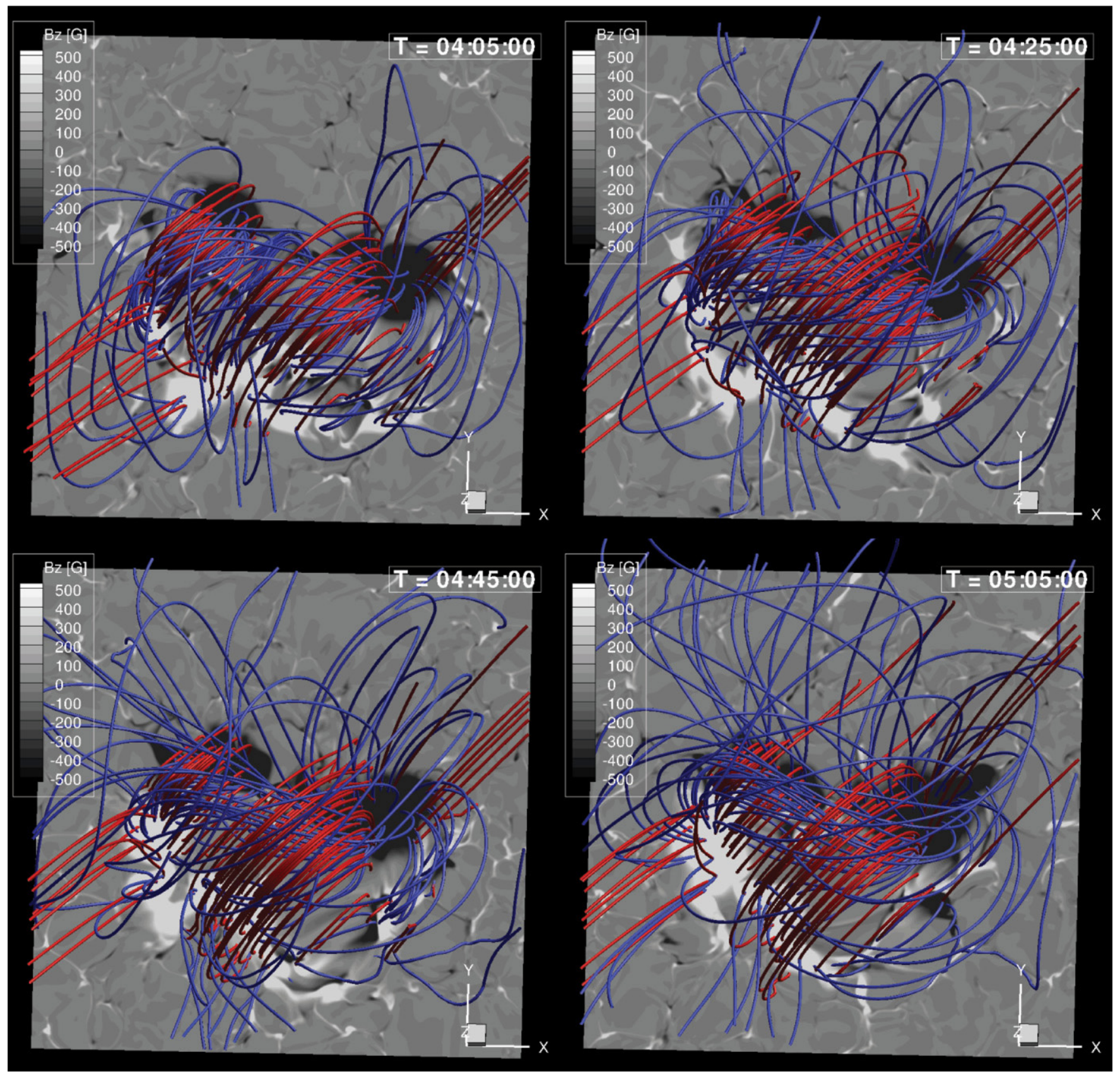

Figure 7. Comparison of the model field (blue) with the extrapolated potential field (red) at time $t=04: 05: 00,04: 25: 00,04: 45: 00$, and 05:05:00. The color on the plane shows the $B_{z}$ field at the photosphere. The clearest departure in field line direction occurs at the sheared field lines above the PIL.

(A color version of this figure is available in the online journal.)

their structure being caused by random convective motions. To get a better understanding of the dynamics, we plot the vertical field strength, flow field, and Poynting fluxes in the low corona at a height of $Z=3 \mathrm{Mm}$ in Figures 5(d)-(f). Here, in the corona, the magnetic field expands and smooths out as the plasma beta drops. This transition allows us to make a much clearer picture of the relevant structures. The flux expands to form two nearby polarities and an elongated PIL between them, shown by Figure 5(d). Here, the presence of a highly sheared field is obvious in the red arrows showing the horizontal field direction. The positive polarity shows little discernible twist while the negative polarity is clearly twisted. Flows are completely dominated by shearing motion at the PIL and rotation in the polarities. Both flows make a significant contribution to the energy transfer, but the energy transported by shear flow tends to be more consistent in time than that of rotational flows.

\section{SUMMARY AND CONCLUSIONS}

Our simulation provides us with a unique opportunity to study an example of magnetic flux emergence in a realistic simulation of the convection zone/coronal system. The system shows a wealth of complexity and interaction of multiple physical processes, which conspire to transport magnetic flux and free energy from the convection zone into the corona. The emerging magnetic flux interacts with convective cells of varying scales as it approaches the photosphere, and then accumulates in convective downdrafts to form a bipolar magnetic structure 

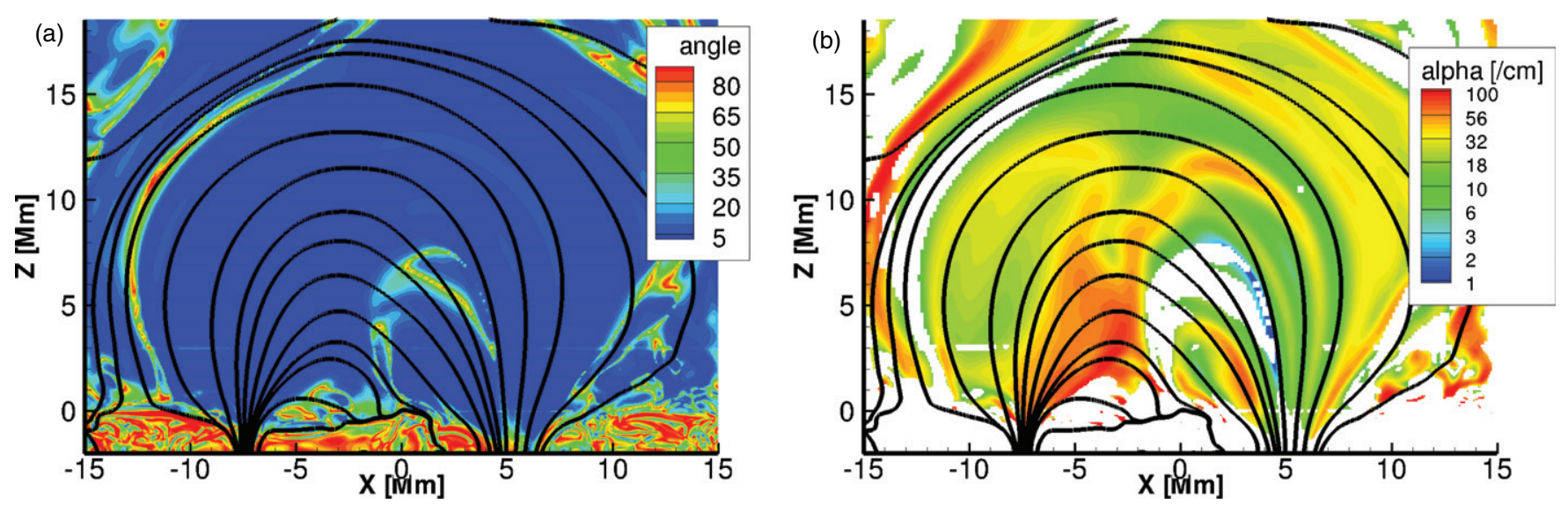

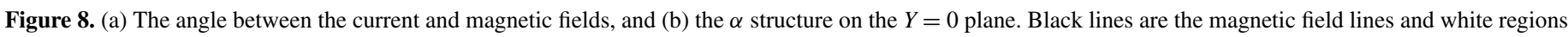
in panel (b) are areas where the angle between the current and magnetic fields is greater than $10^{\circ}$.

(A color version of this figure is available in the online journal.)

resembling a solar active region. The magnetic field continually expands into the upper atmosphere, which results in shear flows and rotating motions that combine to draw the field nearly parallel to the PIL, and transport energy into the corona. At a later time, converging motions at the PIL cause flux cancellation at the photosphere along with tether-cutting reconnection, which produces highly sheared, sigmoid-shaped field lines high in the corona.

In the process of building up the magnetic free energy, shearing flows play a significant role at the photosphere and in the corona. At the near-surface layers, the angle between the current and magnetic fields ranges from $35^{\circ}$ to $90^{\circ}$. The geometry of current and magnetic fields then produces the Lorentz force at the surface, which drives the rotating and shearing motions and increases the non-potentiality of the magnetic fields over a period of hours after the emergence. In particular, the shearing flows align the magnetic fields nearly parallel to the PIL and transport a significant amount of magnetic energy from the convection zone into the corona during the flux emergence, consistent with observations by Schrijver et al. (2005). The shearing flows at the PIL are accompanied by periods of rotating motion of the magnetic polarities. Both of the two motions contribute to the buildup of magnetic shear and free energy in the corona. However, the shearing motions distinguish themselves from the rotation by their persistent presence and concentration at the PIL, where the field has an arcade structure, while rotation, on the other hand, is only present in one of the magnetic polarities and lasts only for $1 \mathrm{hr}$. It is the long-lasting, Lorentz-force-driven shearing motion that dominates the energy transfer.

Convection-driven converging motion drives flux bundles of opposite polarities together, producing a strong magnetic gradient and the pronounced PIL, preferential for large flares (Schrijver 2007; Falconer et al. 2008). The horizontal converging motion at the PIL also contributes to the development of the magnetic shear of the field lines by increasing the shear angle (Martens \& Zwaan 2001). The combination of converging and shearing flows at the PIL forms a group of highly sheared arcades overlying a sharp magnetic PIL. Within this arcade, converging motions lead to the occurrence of tether-cutting reconnection, producing two types of field lines: one long sheared expanding loops and the other unsheared submerging loops. The short loops sink into the convection zone at the speed of convective flows, up to $2 \mathrm{~km} \mathrm{~s}^{-1}$, consistent with Harvey et al.
(1999), which continuously reduces the photospheric unsigned flux. The longer loops rise into the corona with more magnetic shear. The shearing motion along the PIL plays a very important role in that it both produces a sheared arcade structure ready for reconnection and accumulates magnetic shear in the field lines formed after the reconnection. The magnetic configuration of this area thus yields a high free energy up to $40 \%$ of the total, which is comparable with the threshold value for eruptions reported by Amari et al. (2003), Aulanier et al. (2010), and Moore et al. (2012).

Shear flows, converging motions, and tether-cutting reconnection combine to continuously build up the magnetic shear and free energy in the corona necessary for eruptive and explosive events. The magnetic reconnection and shearing motion at the photosphere produce the sigmoid-shaped field geometry, which is preferential for CMEs (Canfield et al. 1999), and the persistent sheared arcade structure is consistent with many CME models (e.g., Steinolfson 1991; Mikic \& Linker 1994; Amari et al. 1996; Guo \& Wu 1998; Antiochos et al. 1999). Furthermore, we find coronal free energy grows at a rate of $3 \times 10^{30} \mathrm{erg} \mathrm{hr}^{-1}$, which builds up $10^{31} \mathrm{erg}$ free energy over $5 \mathrm{hr}$. Such a buildup rate is observed by Schrijver (2007) prior to large flares. Moreover, the majority of the free energy resides low in the corona where it can be confined and later released by reconnection. Finally, we note that the consistent shearing motion and reconnection at the flux cancellation site keep reforming the sigmoid structure, which is essential for homologous eruptive events.

In our simulation here, tether-cutting reconnection and flux cancellation take place in the highly sheared magnetic fields along the PIL, in the presence of converging and shearing flows. In the coupled system of the convection zone, the photosphere, and the corona, all these mechanisms combine to work simultaneously and build up the free magnetic energy in the coronal fields. More importantly, the resulting geometry of the magnetic fields in our domain consists of compact, highly sheared core fields confined by more relaxed, overlying fields. $50 \%$ of the corona free magnetic energy is stored within the compressed, sheared core, while the overlying fields relax very quickly with altitude. The compact core, possessing free magnetic energy up to $10^{30} \mathrm{erg}$, provides the preferential magnetic structure as well as free energy for solar eruptions. We expect that in future simulations of larger scales, shear flows, converging motion, and reconnection will produce enough free magnetic energy and give rise to eruptions of the magnetic system. 
The authors thank Spiro K. Antiochos and Tamas I. Gombosi for constructive discussions and comments. This work was supported by NASA Grants NNG06GD62G, NNX07AC16G and NSF Grants ATM 0642309 and AGS 1023735. W.M. IV was also funded by NASA Grant LWS NNX09AJ78G. W.P.A. was supported in part by NASA LWS TR\&T award NNX08AQ30G, and the Heliophysics Theory Program, under NASA grant NNX08AI56G-04/11. The simulations described here were carried out on the Pleiades system at the NASA Advanced Supercomputing (NAS) Facility and Bluefire cluster at NCAR.

\section{REFERENCES}

Abbett, W. P. 2007, ApJ, 665, 1469

Abbett, W. P., Fisher, G. H., \& Fan, Y. 2001, ApJ, 546, 1194

Amari, T., Luciani, J. F., Aly, J. J., Mikic, Z., \& Linker, J. 2003, ApJ, 585, 1073

Amari, T., Luciani, J. F., Aly, J. J., \& Tagger, M. 1996, ApJ, 466, L39

Antiochos, S. K., DeVore, C. R., \& Klimchuk, J. A. 1999, ApJ, 510, 485

Archontis, V., \& Török, T. 2008, A\&A, 492, L35

Aulanier, G., Török, T., Démoulin, P., \& DeLuca, E. E. 2010, ApJ, 708, 314

Bieber, J. W., \& Rust, D. M. 1995, ApJ, 453, 911

Canfield, R. C., Hudson, H. S., \& McKenzie, D. E. 1999, Geophys. Res. Lett., 26,627

Cheung, M. C. M., Rempel, M., Title, A. M., \& Schüssler, M. 2010, ApJ, 720, 233

Falconer, D. A. 2001, J. Geophys. Res., 106, 25185

Falconer, D. A., Moore, R. L., \& Gary, G. A. 2002, ApJ, 569, 1016

Falconer, D. A., Moore, R. L., \& Gary, G. A. 2006, ApJ, 644, 1258

Falconer, D. A., Moore, R. L., \& Gary, G. A. 2008, ApJ, 689, 1433

Fan, Y. 2001, ApJ, 554, L111

Fan, Y. 2005, ApJ, 630, 543

Fan, Y. 2008, ApJ, 676, 680

Fang, F., Manchester, W., Abbett, W. P., \& van der Holst, B. 2010, ApJ, 714, 1649

Fang, F., Manchester, W., IV, Abbett, W. P., \& van der Holst, B. 2012, ApJ, 745, 37

Forbes, T. G. 2000, J. Geophys. Res., 105, 23153

Forbes, T. G., \& Isenberg, P. A. 1991, ApJ, 373, 294

Forbes, T. G., Linker, J. A., Chen, J., et al. 2006, Space Sci. Rev., 123, 251

Galsgaard, K., Archontis, V., Moreno-Insertis, F., \& Hood, A. W. 2007, ApJ, 666,516

Gopalswamy, N. 2006, Space Sci. Rev., 124, 145

Gosling, J. T., Bame, S. J., McComas, D. J., \& Phillips, J. L. 1990, Geophys. Res. Lett., 17, 901

Green, L. M., Kliem, B., \& Wallace, A. J. 2011, A\&A, 526, A2

Guo, W. P., \& Wu, S. T. 1998, ApJ, 494, 419

Hagyard, M. J., Teuber, D., West, E. A., \& Smith, J. B. 1984, Sol. Phys., 91, 115

Harvey, K. L., Jones, H. P., Schrijver, C. J., \& Penn, M. J. 1999, Sol. Phys., 190, 35

Hundhausen, A. J. 1993, J. Geophys. Res., 98, 13177

Kitiashvili, I. N., Kosovichev, A. G., Wray, A. A., \& Mansour, N. N. 2010, ApJ, 719,307

Leake, J. E., Linton, M. G., \& Antiochos, S. K. 2010, ApJ, 722, 550

Leka, K. D., \& Steiner, O. 2001, ApJ, 552, 354
Li, Y., Luhmann, J., Fisher, G., \& Welsch, B. 2004, J. Atmos. Sol.-Terr. Phys., 66,1271

Linker, J. A., Mikić, Z., Lionello, R., et al. 2003, Phys. Plasmas, 10, 1971

Lites, B. W. 2005, ApJ, 622, 1275

Lites, B. W., Low, B. C., Martinez Pillet, V., et al. 1995, ApJ, 446, 877

Liu, C., Deng, N., Liu, Y., et al. 2005, ApJ, 622, 722

Low, B. C. 1994, Phys. Plasmas, 1, 1684

Low, B. C. 2001, J. Geophys. Res., 106, 25141

Luoni, M. L., Démoulin, P., Mandrini, C. H., \& van Driel-Gesztelyi, L. 2011, Sol. Phys., 270, 45

Lynch, B. J., Antiochos, S. K., DeVore, C. R., Luhmann, J. G., \& Zurbuchen, T. H. 2008, ApJ, 683, 1192

Lynch, B. J., Antiochos, S. K., MacNeice, P. J., Zurbuchen, T. H., \& Fisk, L. A. 2004, ApJ, 617, 589

Lynch, B. J., Li, Y., Thernisien, A. F. R., et al. 2010, J. Geophys. Res. (Space Phys.), 115, A07106

MacTaggart, D., \& Hood, A. W. 2009, A\&A, 507, 995

Magara, T., \& Longcope, D. W. 2003, ApJ, 586, 630

Manchester, W. 2008, in ASP Conf. Ser. 383, Subsurface and Atmospheric Influences on Solar Activity, ed. R. Howe, R. W. Komm, K. S. Balasubramaniam, \& G. J. D. Petrie (San Francisco, CA: ASP), 91

Manchester, W., IV 2001, ApJ, 547, 503

Manchester, W., IV 2007, ApJ, 666, 532

Manchester, W., IV, Gombosi, T., DeZeeuw, D., \& Fan, Y. 2004, ApJ, 610, 588

Martens, P. C., \& Zwaan, C. 2001, ApJ, 558, 872

Martin, S. F., Livi, S. H. B., \& Wang, J. 1985, Aust. J. Phys., 38, 929

Matsumoto, R., Tajima, T., Shibata, K., \& Kaisig, M. 1993, ApJ, 414, 357

Mikic, Z., \& Linker, J. A. 1994, ApJ, 430, 898

Moore, R. L., Falconer, D. A., \& Sterling, A. C. 2012, ApJ, 750, 24

Moore, R. L., Sterling, A. C., Hudson, H. S., \& Lemen, J. R. 2001, ApJ, 552, 833

Parker, E. N. 1977, ARA\&A, 15, 45

Rachmeler, L. A., DeForest, C. E., \& Kankelborg, C. C. 2009, ApJ, 693, 1431

Rempel, M. 2011, ApJ, 729, 5

Rempel, M., Schüssler, M., \& Knölker, M. 2009, ApJ, 691, 640

Rogers, F. J. 2000, Phys. Plasmas, 7, 51

Roussev, I. I., Sokolov, I. V., Forbes, T. G., et al. 2004, ApJ, 605, L73

Schrijver, C. J. 2007, ApJ, 655, L117

Schrijver, C. J. 2009, Adv. Space Res., 43, 739

Schrijver, C. J., De Rosa, M. L., Metcalf, T., et al. 2008, ApJ, 675, 1637

Schrijver, C. J., De Rosa, M. L., Title, A. M., \& Metcalf, T. R. 2005, ApJ, 628, 501

Shibata, K., Tajima, T., Steinolfson, R. S., \& Matsumoto, R. 1989, ApJ, 345, 584

Spruit, H. C., \& van Ballegooijen, A. A. 1982, A\&A, 106, 58

St. Cyr, O. C., Plunkett, S. P., Michels, D. J., et al. 2000, J. Geophys. Res., 105 , 18169

Stein, R. F., \& Nordlund, Å. 2006, ApJ, 642, 1246

Steinolfson, R. S. 1991, ApJ, 382, 677

Sturrock, P. A., Weber, M., Wheatland, M. S., \& Wolfson, R. 2001, ApJ, 548, 492

Su, Y., Golub, L., \& Van Ballegooijen, A. A. 2007, ApJ, 655, 606

Subramanian, P., \& Dere, K. P. 2001, ApJ, 561, 372

Titov, V. S., \& Démoulin, P. 1999, A\&A, 351, 707

Tokman, M., \& Bellan, P. M. 2002, ApJ, 567, 1202

Török, T., \& Kliem, B. 2005, ApJ, 630, L97

van der Holst, B., Manchester, W., IV, Sokolov, I. V., et al. 2009, ApJ, 693, 1178

Yang, G., Xu, Y., Cao, W., et al. 2004, ApJ, 617, L151

Zhang, J., Dere, K. P., Howard, R. A., \& Vourlidas, A. 2004, ApJ, 604, 420

Zirin, H., \& Wang, H. 1993, Nature, 363, 426 